\title{
Short-period variability in the galactic cosmic ray intensity: High statistical resolution observations and interpretation around the time of a Forbush decrease in August 2006
}

\author{
T. Mulligan, ${ }^{1}$ J. B. Blake, ${ }^{1}$ D. Shaul, ${ }^{2}$ J. J. Quenby,${ }^{2}$ R. A. Leske, ${ }^{3}$ R. A. Mewaldt,${ }^{3}$ \\ and M. Galametz ${ }^{2,4}$ \\ Received 1 October 2008; revised 21 January 2009; accepted 16 March 2009; published 21 July 2009.
}

[1] On 20 August 2006 a Forbush decrease observed at Polar in the Earth's magnetosphere was also seen at the INTEGRAL spacecraft outside the magnetosphere during a very active time in the solar wind. High-resolution energetic particle data from ACE SIS, the Polar high-sensitivity telescope, and INTEGRAL's Ge detector saturation rate, which measures the galactic cosmic ray (GCR) background with a threshold of $\sim 200 \mathrm{MeV}$, show similar, short-period GCR variations in and around the Forbush decrease. Focusing upon the GCR intensity within a 3-day interval from 19 August 2006 to 21 August 2006 reveals many intensity variations in the GCR on a variety of time scales and amplitudes. These intensity variations are greater than the $3 \sigma$ error in all the data sets used. The fine structures in the GCR intensities along with the Forbush decrease are propagated outward from ACE to the Earth with very little change. The solar wind speed stays relatively constant during these periods, indicating that parcels of solar wind are transporting the GCR population outward in the heliosphere. This solar wind convection of GCR fine structure is observed for both increases and decreases in GCR intensity, and the fine structure increases and decreases are bracketed by solar wind magnetic field discontinuities associated with interplanetary coronal mass ejection (ICME) magnetosheath regions, clearly seen as discontinuous rotations of the field components at $\mathrm{ACE}$ and at Wind. Interestingly, the electron heat flux shows different flux tube connectivity also associated with the different regions of the ICME and magnetosheath. Gosling et al. (2004) first discussed the idea that solar energetic particle intensities commonly undergo dispersionless modulation in direct association with discontinuous changes in the solar wind electron strahl. The observations show that the intensity levels in the GCR flux may undergo a similar partitioning, possibly because of the different magnetic field regions having differing magnetic topologies.

Citation: Mulligan, T., J. B. Blake, D. Shaul, J. J. Quenby, R. A. Leske, R. A. Mewaldt, and M. Galametz (2009), Short-period variability in the galactic cosmic ray intensity: High statistical resolution observations and interpretation around the time of a Forbush decrease in August 2006, J. Geophys. Res., 114, A07105, doi:10.1029/2008JA013783.

\section{Introduction}

[2] The research of Scott Forbush involved the detailed study of the temporal variations in the intensity of cosmic rays. One such variation is now named for him, a Forbush decrease (FD) being a global transient decrease in cosmic ray intensity followed by a substantially slower recovery.

\footnotetext{
${ }^{1}$ Aerospace Corporation, Los Angeles, California, USA.

${ }^{2}$ High Energy Physics Group, Blackett Laboratory, Imperial College London, London, UK.

${ }^{3}$ California Institute of Technology, Pasadena, California, USA.

${ }^{4}$ Now at Laboratoire AIM, DSM, CEA, Université Paris Diderot, DAPHNE, Service d'Astrophysique, CNRS, Gif-sur-Yvette, France.

Copyright 2009 by the American Geophysical Union. 0148-0227/09/2008JA013783\$09.00
}

Since Forbush's discovery and description of these phenomena in the late 1930s, FDs have been put into context with increasing developments within heliospheric physics. In particular, detailed observations of coronal mass ejections (CMEs), their interplanetary counterparts (ICMEs), and in situ observations of the solar wind and energetic particles have greatly increased understanding of the underlying physics of FDs. Comprehensive review articles on FDs were written by Lockwood [1971], and later by Cane [2000] and Richardson [2004]. Much work has been done to establish a relationship between energetic particle variability and transient phenomena causing FDs. Outside of the aforementioned review papers, Richardson et al. [2000] discuss bidirectional flows of energetic ions at GCR energies, relating these to substructures in ICMEs such as different flux tubes within the ejecta. Typical of 
studies found in the literature, their observations were limited to observations greater than $2 \mathrm{~h}$ in duration because of the resolution limitations of their measurements. Vasquez et al. [2001] performed a similar study focusing on the possibility that Alfvén waves near tangential discontinuities could cause changes in the GCR flux intensity. Again, these observations were limited by the time resolution of their data. In both papers the authors speculate that the boundaries of substructures within ICMEs may be topological, separating different regions of plasma and fields, but neither paper expounds on this idea.

[3] Similar to Richardson et al. [2000], many authors have also used neutron monitor networks such as "Spaceship Earth" or other ground-based particle detectors to draw associations between high-energy particle anisotropies with ICME magnetic structure and substructures [Bieber and Evenson, 1995; Munakata et al., 2006]. In particular, Kuwabara et al. [2008] discuss the high-energy anisotropy of the FD during the time interval studied in this paper using the newly constructed IceTop air shower array. Unfortunately, these observations cannot be directly applied to the current study as our secondary, high count rate measurements have no understandable anisotropy sensitivity.

[4] In this paper, we concentrate on smaller amplitude and higher-frequency variability in the galactic cosmic ray (GCR) intensity than represented by the classical FD. Our focus is on variability on time scales of a few hours or less. One of the authors, D. A. Shaul, is involved in several fundamental physics missions, including LISA [Sumner and Shaul, 2004] and ASTROD [Ni et al., 2006], which rely on the realization of a high precision of geodesic motion of an isolated test mass in space, primarily over a frequency range of $0.1-0.1 \mathrm{mHz}$. These test masses are subject to charging by the impinging GCR, resulting in spurious forces. Nominally, the test masses will be discharged using UV light, thus limiting the disturbances associated with charging. However, the level to which disturbances can be minimized will be determined by how well the charging rate can be tracked. The present study is part of a series aimed at determining the variability in flux in the frequency range of interest for these missions to enable optimization of charge management techniques.

[5] Small-amplitude, $\mathrm{mHz}$ variability in the GCR is an experimental challenge in that very large instrumental geometric factors are required in order to make statistically significant measurements of the GCR in time periods of a few minutes or less. Undoubtedly, this is a major factor in the lack of literature about such variability on $\mathrm{mHz}$ time scales. In this paper, spacecraft instrumentation that was not designed for GCR research, but for which the GCR are considered background events that must be removed from the data in order to do the primary science, will be used, in particular, data from the SPI instrument aboard the INTEGRAL gamma ray observatory. The huge size of the detectors in SPI, and of the spacecraft itself (a source of GCR-induced secondaries), leads to high count rates from the GCR.

[6] The paper is organized as follows: salient details of the spacecraft and instrumentation will be discussed in section 2. Details of the event will be described in section 3 . Interpretation of the data and its implications on existing and future studies will be addressed in the discussion and conclusions.

\section{Spacecraft and Instrumentation}

[7] Data from INTEGRAL, Polar, and ACE are used for this study. We describe the INTEGRAL and Polar spacecraft and instrumentation only insofar as they are used to measure the time history of the intensity of the GCR. INTEGRAL and Polar do not have GCR studies as a science objective. Instead, these two missions are employed as sensors of opportunity, using as primary data what are only considered background events for the prime mission objectives.

[8] In contrast to the cases of INTEGRAL and Polar, the primary mission of the ACE spacecraft is to measure energetic particles from a variety of sources, including those in the GCR population. However, the instrumentation is not focused upon measuring protons with energies of a few hundred $\mathrm{MeV}$ and above so in that sense the GCR population of interest in this paper is also a background population. Although an unusual use of particle instrumentation, this work certainly is not the first time energetic particle sensors designed for other applications are to be used to observe the temporal history of GCR populations. Richardson [2004] in a major review paper discusses several examples of using anticoincidence guards as a primary source of GCR data, as do McCracken et al. [1966], Richardson et al. [1996], and Vasquez et al. [2001]. We shall return to the topic of the effectiveness of such earlier measurements to detect shortterm variability in the GCR flux after describing the instrumentation used in this paper. The ESA gamma ray observatory, INTEGRAL, launched on 17 October 2002, has an orbit with apogee $\sim 24 \mathrm{RE}$, perigee $\sim 1.4 \mathrm{RE}$, and inclination of $51.6^{\circ}$. The orbital period is 3 days. The high apogee is chosen to minimize the time INTEGRAL spends traversing the Earth's radiation belts.

[9] One of the experiments aboard INTEGRAL is the SPI spectrometer, consisting of an array of 19 actively cooled germanium detectors. Each Ge detector is hexagonal in shape, with a side length of $3.2 \mathrm{~cm}$ and a height of $7 \mathrm{~cm}$ [Vedrenne et al., 2003]. The Ge detectors are surrounded by an anticoincidence shield (ACS) made up of 91 bismuth germanate oxide (BGO) blocks. Cosmic rays interact directly with the Ge detectors, or with materials on the spacecraft, producing a detectable background that varies because of modulations in the incident cosmic ray flux. The magnitude of this variation is typically of the same order of magnitude as the $511-\mathrm{keV}$ gamma ray signal from the Galactic center, a primary INTEGRAL observation goal [Teegarden et al., 1997]. Consequently, the INTEGRAL SPI detectors are excellent monitors of the GCR flux, and as such they are used in the present work to study the shortterm GCR flux variability. These same measurements are used by the INTEGRAL experimenters to ensure that background variations due to cosmic rays may be reliably subtracted from the gamma ray signal [cf. Teegarden et al., 1997; Jean et al., 2003].

[10] Methods available for GCR detection include the saturated count rates in the ACS (ACSSAT) and the Ge detector system (GEDSAT). In both cases, "saturated" is taken to mean the amplitude saturation of the detector 


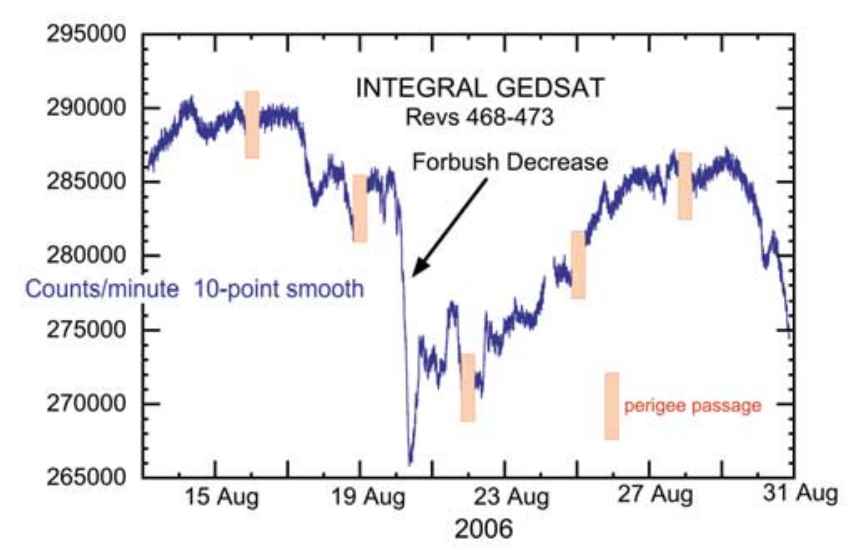

Figure 1. The GEDSAT data from INTEGRAL over the time period from rev 468 through rev 473 is plotted. The time period shown is from 13 August 2006 through 30 August 2006. The bars show perigee passage and therefore times when no GEDSAT data are available.

system. The Ge detectors saturate at $\sim 10 \mathrm{MeV}$, resulting in an effective GEDSAT cosmic ray threshold of $\sim 200 \mathrm{MeV}$, a consequence of the energy required to penetrate the spacecraft shielding and reach the Ge detectors with enough energy remaining to deposit at least $10 \mathrm{MeV}$ [Teegarden et al., 1997]. The ACSSAT threshold is $\sim 150 \mathrm{MeV}$ [Jean et al., 2003]. The ACS count rate is also available and has a low threshold of $75 \mathrm{keV}$ [Vedrenne et al., 2003]. Even though the ACS rates have even better statistics than the GEDSAT rates, they also appear to have a significant response to GCR secondaries such as neutrons, so we will limit ourselves to using the GEDSAT rates only for this study.

[11] Figure 1 shows the GEDSAT count rate over six orbits (revs) of the INTEGRAL spacecraft. The FD is the largest intensity change during the six revs, but there are many others with differing amplitudes that occur on a variety of time scales. Note the GEDSAT count rates reveal a wealth of statistically significant detail, which is enabled by the very high count rates and large statistics.

[12] As a final note, the INTEGRAL Radiation Environment Monitor (IREM) is used to determine when the spacecraft is within the radiation belts [cf. Hajdas et al., 2003]. Although it is used for checking that the GEDSAT rates are not contaminated by radiation belt particles, IREM is not suitable for the high-statistics GCR observations required for this study.

[13] The NASA Polar spacecraft (1996-013A) was launched on 24 February 1996 into an orbit with an inclination of $86^{\circ}$, an apogee of $9 \mathrm{RE}$, and a perigee of $1.8 \mathrm{RE}$ geocentric. Initially, apogee was at high northern latitudes, but since launch, apogee has slowly moved $(\sim 16 \%)$ to the equator, and then to high southern latitudes. For the present study, we discuss measurements made in the summer of 2006 when the satellite apogee was over the southern polar cap.

[14] One of the twelve investigations aboard Polar is CEPPAD (Comprehensive Energetic Particle Pitch Angle Distribution) [cf. Blake et al., 1995]. CEPPAD contains three detector systems; the one of interest for GCR studies is called HIST (high-sensitivity telescope) and is described in detail in a thesis by Contos [1997]. The primary purpose of
HIST is to measure energy spectra and angular distributions of energetic radiation belt electrons in the energy range from $\sim 1 \mathrm{MeV}$ to $10 \mathrm{MeV}$. A secondary objective is to measure the proton population from $\sim 5 \mathrm{MeV}$ to $100 \mathrm{MeV}$. HIST is a telescope consisting of three detectors. The first two detectors are silicon disks with thicknesses of $324 \mu \mathrm{m}$ and $2000 \mu \mathrm{m}$, respectively. The third detector is a plastic scintillator viewed from behind by a photomultiplier tube. The shape and volume of the scintillator was determined by the goal of containing the energy deposit of incident electrons up to $10 \mathrm{MeV}$.

[15] In this paper, HIST is not used in its primary mode as a particle telescope. Instead, we measure only the count rate in the plastic scintillator, using the scintillator as an omnidirectional sensor. The omnidirectional geometric factor of the scintillator is $465 \mathrm{~cm}^{2}$ sr [Contos, 1997]. The amplitude of each event in the scintillator is digitized into 256 channels (channel 0 to channel 255) from $\sim 100 \mathrm{keV}$ to $10 \mathrm{MeV}$. All pulses larger than $10 \mathrm{MeV}$ go into Channel 255, the "overflow" channel. The plastic scintillator is sufficiently large such that most GCR particles deposit well over $10 \mathrm{MeV}$. Therefore, primary GCR events appear mainly in Channel 255, with exceptions being "corner cutters" and particles that just reach the scintillator at end of range. The energy response of the overflow channel has been determined to be $\sim 100 \mathrm{MeV}$ through cross calibration with the GOES 11 proton instrument during the solar energetic particle event on 13 December 2006 [Mulligan et al., 2008]. During any particular orbit, it should be noted that passages into the Earth's radiation belt are easily seen. These radiation belt passages differ significantly in detail from one another, but the presence of magnetospheric particles is readily identified, and those time periods are removed for the present study. The orbit of Polar is such that approximately two thirds of the time it is outside of the radiation belts where the only energetic particles to be found are the GCR (excluding energetic solar particle events). The only response of HIST during these times is a "background" count rate caused by the GCR. In this regard, Polar and INTEGRAL are similar.

[16] The ACE observatory is located at the Sun-Earth L1 libration point at $240 \mathrm{Re}$ sunward of the Earth. The spacecraft spins with the spin axis pointed nominally toward the Sun. The prime objective of ACE is to determine and compare the elemental and isotopic composition of several distinct samples of matter, including the solar corona, the interplanetary medium, the local interstellar medium, and galactic matter. One of several instruments aboard ACE is the Solar Isotope Spectrometer (SIS), designed to provide high-resolution measurements of the isotopic composition of energetic nuclei from $\mathrm{He}$ to $\mathrm{Ni}(\mathrm{Z}=2$ to 28$)$ over the energy range from $\sim 10$ to $\sim 100 \mathrm{MeV} /$ nucleon [Stone et al., 1998]. In order to maximize the number of detected particles for both the GCR flux and the solar energetic particles (SEPs), SIS has two telescopes composed of the largest area devices available at the time of instrument development $\left(\sim 65 \mathrm{~cm}^{2}\right.$ each). The SIS geometric factor is $\sim 40 \mathrm{~cm}^{2}$ sr, with each of the SIS detector stacks consisting of 17 silicon detectors [cf. Stone et al., 1998].

[17] For present purposes, we use only the singles counting rate in detector T8. For protons entering the telescope through the aperture, the threshold energy is $\sim 40 \mathrm{MeV}$ and 


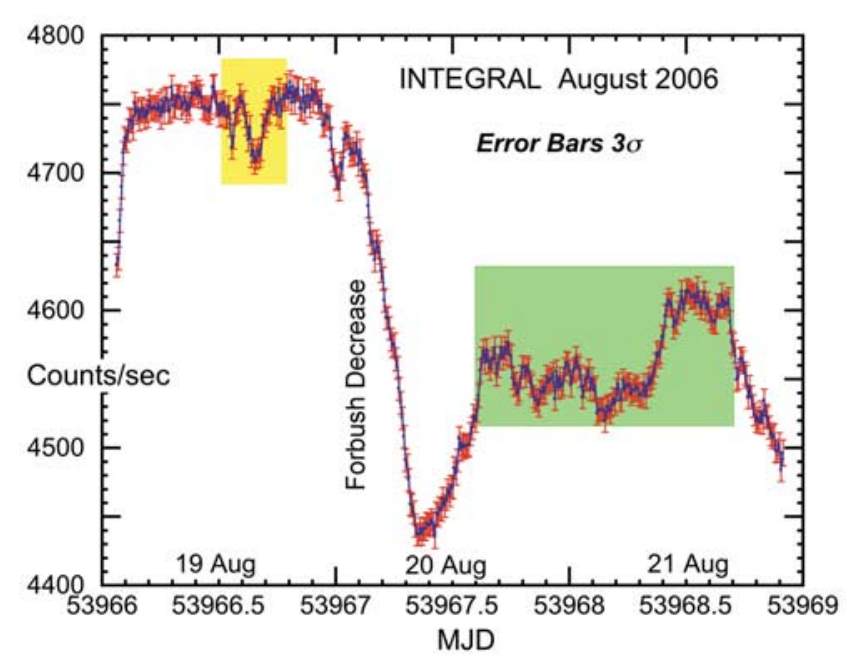

Figure 2. The GEDSAT count rate from INTEGRAL is plotted for an entire orbit of approximately 3 days. The rise in count rate at the beginning of the plot is due to the INTEGRAL spacecraft moving beyond the geomagnetic cutoff. The data points have $3 \sigma$ error bars. The highlighted regions (yellow and green) are the two intervals of GCR flux variability under study, which occur beyond the main Forbush decrease.

extends to $\sim 100 \mathrm{MeV}$. Response through the sides of the box starts at $\sim 100 \mathrm{MeV}$. Because the GCR energy spectrum peaks at several hundred $\mathrm{MeV}$, it should be noted that most of the counts during quiet times (e.g., no SEPs) will come from particles that have sufficient range to penetrate the stack from outside the telescope field of view. The situation is analogous to that for the sensors aboard INTEGRAL and Polar.

[18] In summary, the sensors aboard INTEGRAL, Polar, and $\mathrm{ACE}$ measure the GCR as penetrating background counts in instruments designed for other purposes. The three sensors are able to detect most of the GCR population with complex energy thresholds that depend upon the direction of arrival of the GCR. For the study described in this paper, detailed energy responses are not needed. Rather, we take advantage of the large geometric factors that all three sensors have for penetrating particles and use the resulting large counting rates to study the short-term variability of the GCR.

[19] To return briefly to the topic of earlier GCR observations, our measurements differ from earlier work described by Richardson [2004] in that we have higher sampling rates in the case of the Polar and INTEGRAL sensors in addition to having large geometric factors. For example the Polar HIST channel counts at $\sim 130$ counts/s and is sampled every 1.5 seconds. The INTEGRAL GEDSAT channel is counting almost 5000 counts/s and is sampled every second. These features of the Polar HIST, and especially the INTEGRAL instrumentation, offer the opportunity to observe GCR variability over shorter time periods than heretofore described.

[20] The ACE-SIS rate is nearly the same as the Polar rate, but is only sampled once every $256 \mathrm{~s}$. Sampling at such a low rate is typical for background channels from instruments focused on studying SEPs, especially from distant spacecraft such as ACE, because of telemetry limitations.

[21] By comparison, Richardson et al. [1996] (particularly their Figures 2 and 3) give count rates without indicating the time over which the counts were collected. However, their plots suggest an integration period of no shorter than an hour. Vasquez et al. [2001] do reveal an IMP 8 count rate of $\sim 460$ counts/s. Their plots indicate an integration period of about an hour. Because the authors do not focus on the details of the GCR measurements, it is not clear whether the background data are summed over several minutes, and the sum telemetered, or whether a snapshot of the background rate is taken.

\section{Observations}

[22] In section 2, we showed six orbits of the GEDSAT count rates from INTEGRAL. We now focus upon the
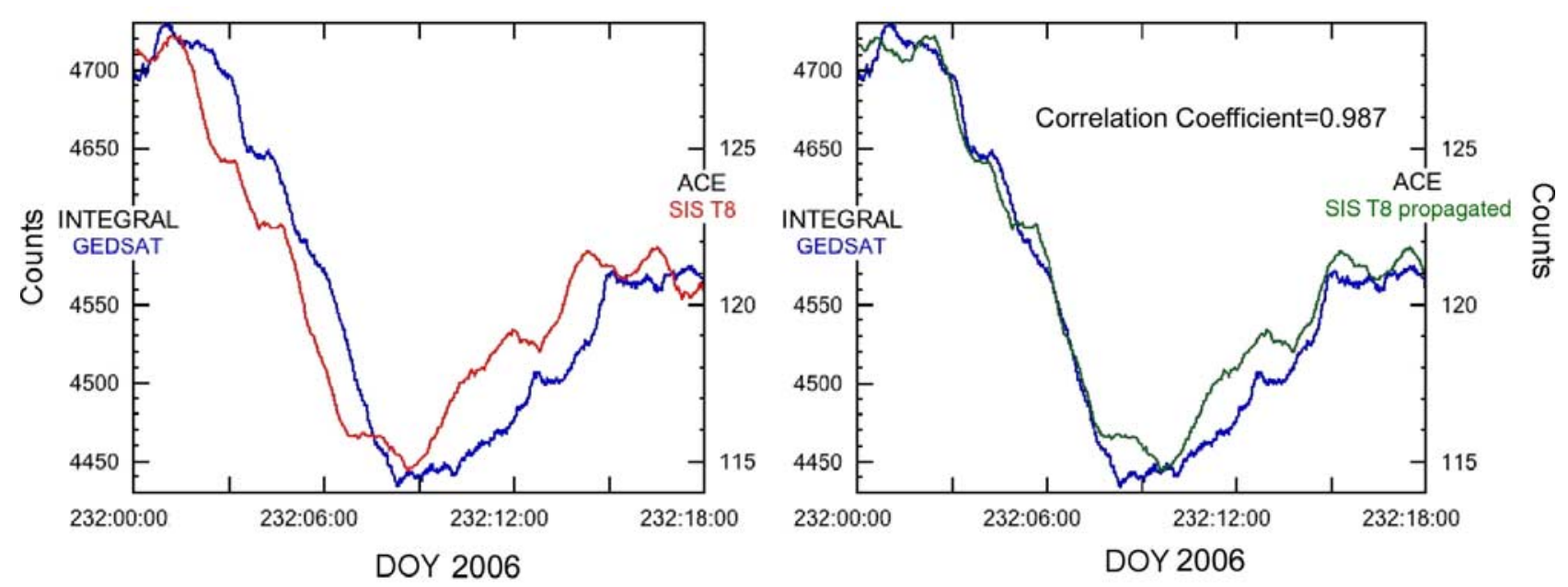

Figure 3. (left) The INTEGRAL/GEDSAT and the ACE/SIS T8 count rates as a function of time during the Forbush decrease on 20 August 2006 (day 232 of 2006). (right) The same count rates with the ACE measurements delayed by the solar wind propagation time from ACE to INTEGRAL, with a correlation coefficient of 0.987 . 


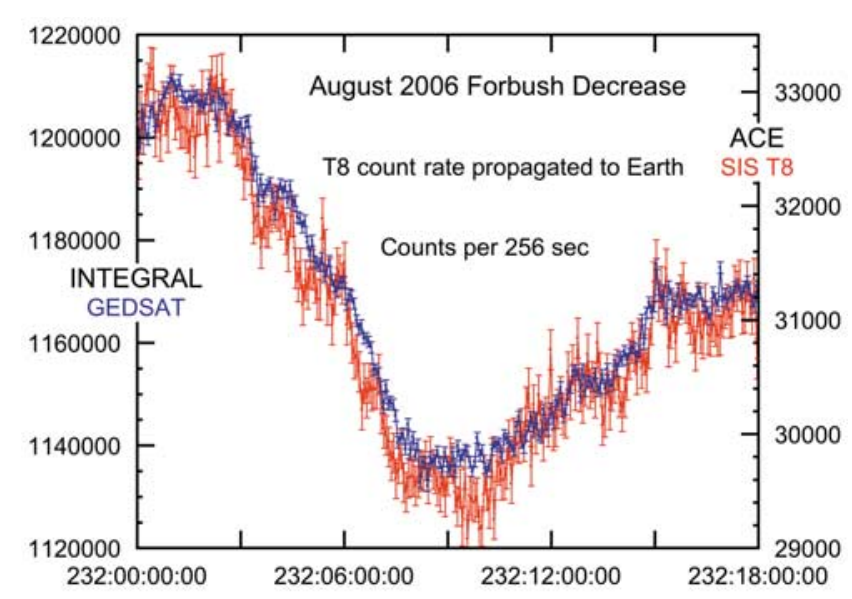

Figure 4. The count rates, 256-s sums, during the time period of the Forbush decrease as seen at ACE (SIS T8) at L1 and INTEGRAL (GEDSAT) at Earth, are plotted together. The ACE count rate has been time delayed by the propagation time to Earth using the measured solar wind parameters. The vertical scales of both count rates have been adjusted to make them overlay.

single INTEGRAL orbit that contains the FD. The data for this orbit are shown in Figure 2. Although the FD is by far the largest variation in count rate, many other count rate structures are clearly seen. Note that the error bars plotted in Figure 2 are $3 \sigma$ to emphasize the fact that the smaller structures are not statistical effects. In addition to the FD, we will examine the count rate variations during the time periods delineated by the highlighted boxes (yellow and green boxes).

[23] We begin with the time period of the Forbush decrease. The GEDSAT and SIS T8 rates are plotted in the Figure 3 (left) on the same time scale, with the ordinate (count rate) adjusted so as to overlap. The SIS data shown in the plot are the average count rate over $256 \mathrm{~s}$, which have been further smoothed using a 10-point running average. The GEDSAT data are sums over $60 \mathrm{~s}$, with a 20-point running average. Both data sets have been smoothed to remove statistical variability and aid the eye in making a quick comparison. The magnitude of the GEDSAT decrease is $\sim 6.9 \%$, whereas for SIS T8, the decrease is $\sim 11.7 \%$. A difference is expected because of the different energy thresholds in the two instruments.

[24] In Figure 3 (right), the ACE data have been plotted, time delayed by the propagation time from ACE at L1 to INTEGRAL near the Earth. The time delay is calculated using the average solar wind speed of $410 \mathrm{~km} / \mathrm{s}$ over this interval. Although the solar wind magnetic field shows this is a very active time in the solar wind, (see Figure 11) the solar wind plasma speed stays reasonably steady at $\sim 410 \mathrm{~km} / \mathrm{s}$ during the interval, simplifying the calculation of a convective time delay to Earth. Note in Figure 3 (right) that after the convective time delay is added, the time history profiles at ACE and INTEGRAL are very similar, with a correlation coefficient of better than 0.98 . It seems clear that a parcel of solar wind moved outward during this time, transporting the GCR population containing the Forbush decrease within it. The position of ACE at the onset is $\mathrm{XGSE}=1.45 \times 10^{6} \mathrm{~km}$, nominally at L1. A measure of the minimum possible cross section of the region containing the $\mathrm{FD}$ is indicated by the other two coordinates of ACE, YGSE $=1.50 \times 10^{5} \mathrm{~km}$, and ZGSE $=$ $3.52 \times 10^{4} \mathrm{~km}$.

[25] In order to show the statistical uncertainties, the smoothed data in Figure 3 has been redone in Figure 4, without smoothing, using 256-s sums of the count rates and with the error bars shown for each point. The ACE count rate has been propagated to Earth using the same delay as in Figure 3. The plot makes clear that the fine structure in the FD is propagated from ACE to Earth with very little change.

[26] At the time of the FD, INTEGRAL was at high northern latitudes and in front of the Earth. Polar was at high altitude over the southern polar cap. Thus, both spacecraft were well separated and outside of the radiation belts. Figure 5 is a plot of INTEGRAL and Polar data showing the onset of the FD. Note that the count rate profile of the Polar HIST instrument is similar to that of the INTEGRAL GEDSAT sensor. The overall agreement is excellent, but there are short time period differences. In this limited run of data, it is difficult to separate statistical fluctuations from the small differences in the variability of the GCR between INTEGRAL and Polar. However, one can say that the presence of the nearby Earth did not have a marked effect.

[27] Figure 6 shows the INTEGRAL data during the $12-\mathrm{h}$ period highlighted by the first box (yellow box) in Figure 2. Statistically significant variability is obvious, and the time profile is quite different from that of the FD. Note the rise and fall time are much more symmetric for this smaller transient.

[28] In Figure 7, the INTEGRAL and ACE data are plotted for the same highlighted interval, using the same format as that used in Figure 3. As is the case for the period of the FD, the temporal history is very similar at both INTEGRAL and ACE. When the ACE count rate is delayed by the solar wind flight time between ACE and the Earth, the congruence between the count rate profiles is striking, having a correlation coefficient of 0.78 . Again, it appears that fine structure in the GCR intensities is being

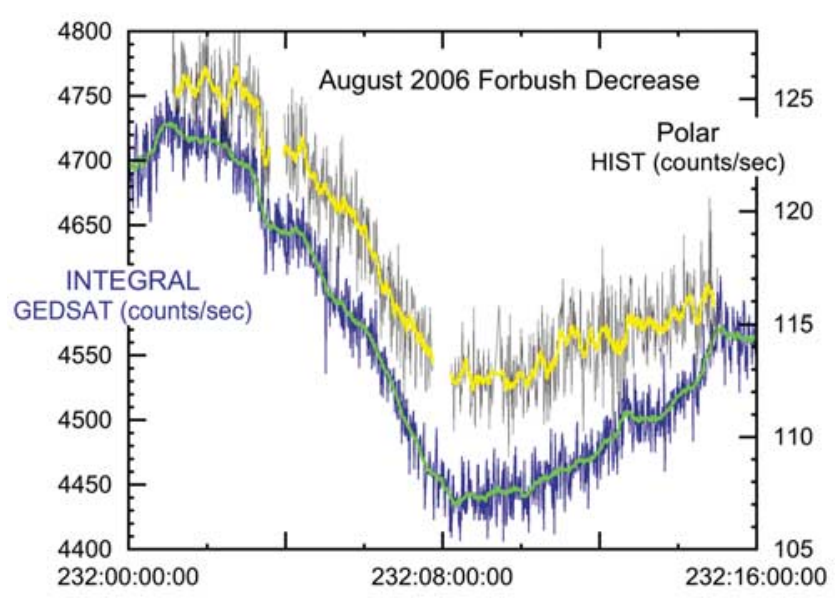

Figure 5. The INTEGRAL/GEDSAT and the Polar HIST count rates are plotted on the same time scale. The Polar data shows the entire time period that Polar was around apogee and outside of the radiation belts. 


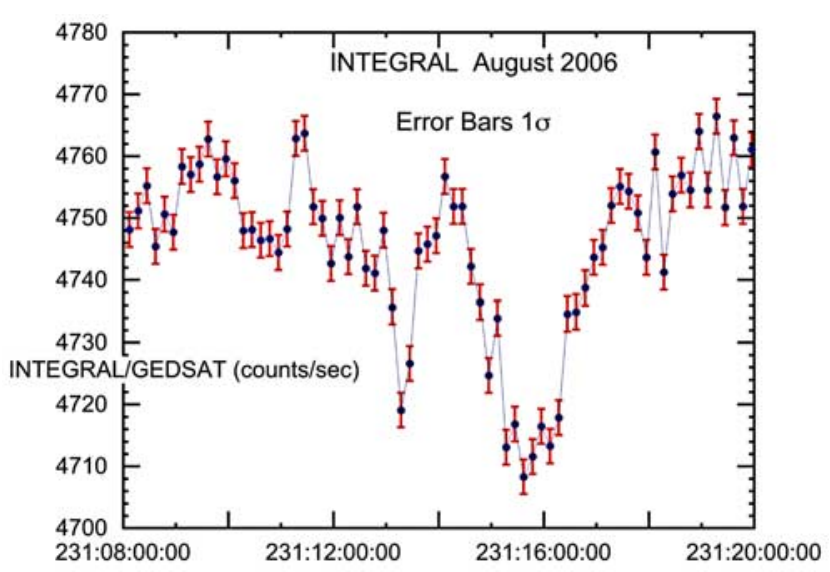

Figure 6. The INTEGRAL/GEDSAT data are plotted for the 12-h period shown in Figure 2, the first (in time) highlighted box.

transported outward in the solar wind. As was done for the case of the FD, a comparison of unsmoothed ACE and INTEGRAL data is shown in Figure 8 to illustrate the statistical uncertainties. This transient is smaller than the FD so the statistical variability is more prominent. In spite of this, the similarity in the time history is great during the first half of the time period. Although it becomes poorer in the latter half, it is clear that the short-period variability is not due solely to changes in the GCR population on a large scale, but is also due to small-scale intensity structures being transported outward by the solar wind.

[29] The time period indicated by the second highlighted box (green box) in Figure 2 goes from the end of the FD recovery until shortly before the INTEGRAL spacecraft was shut down on rev 470 for radiation belt passage. Figure 9 shows the count rate at ACE and INTEGRAL for this interval, with the ACE count rate delayed by the time required for the solar wind to propagate from L1 to Earth.

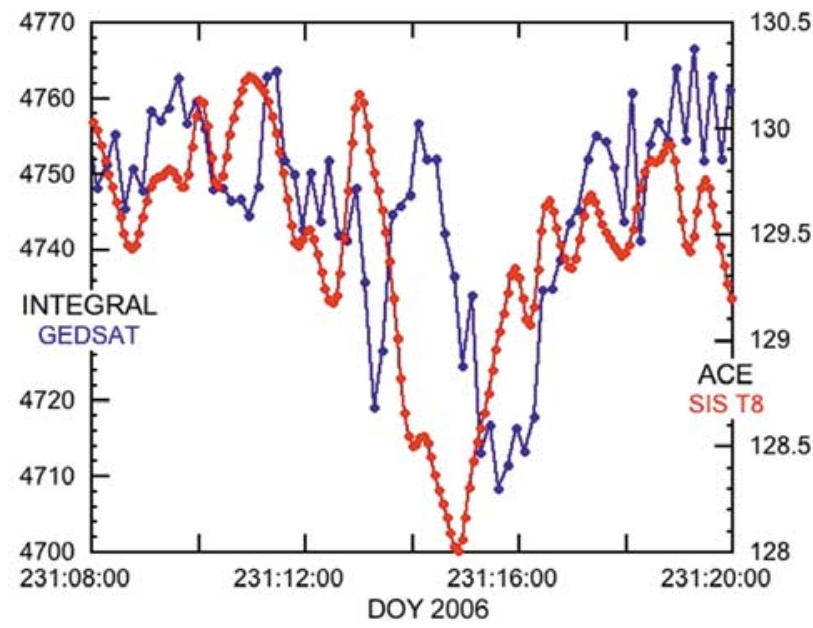

The congruence, as in the earlier examples, is extremely good in general, including the fine structure. This time interval includes enhancements as well as decreases in the GCR count rate, meaning that solar wind "parcels" contain increased GCR intensities as well as decreases. The interval delineated by the gray box indicates a time when the ACE/INTEGRAL count rates differ significantly, with the ACE data showing an apparent oscillatory behavior not seen by INTEGRAL. Figure 10 shows this time period, encompassing the gray box, on an expanded scale.

\section{Discussion}

[30] In section 3, we focused upon the single INTEGRAL orbit of approximately 3 days, containing the Forbush decrease, and thoroughly examined the count rate variations during the Forbush decrease and during the highlighted intervals (Figure 2). In this section, we will concentrate on the smaller decreases within the highlighted intervals. We will put these particle observations in context with the solar wind variability during this period, and discuss the correlations with both large-scale and small-scale solar wind transient structures.

[31] On 20 August 2006, the Forbush decrease observed at Polar and INTEGRAL occurred during the passage of a complex interplanetary coronal mass ejection during an unusually active period in the solar wind. Figure 11 shows the solar wind during a 2-week interval bracketing the Forbush decrease. The first four panels show the $B_{x}, B_{y}$, and $B_{z}$ components in GSE coordinates, and the scalar field $\left(B_{t}\right)$, respectively, from both $\mathrm{ACE}$ (red trace) and Wind (blue trace). The fifth to seventh panels show the proton density, speed, and temperature of the bulk plasma. The GEDSAT energetic particle data from INTEGRAL is plotted in the last panel. Looking at the magnetic field and plasma conditions during this time reveals several interplanetary shocks present in the days leading up to and including the Forbush decrease. Note that there are two interplanetary shocks present during this period (labeled S1, S2) as well as

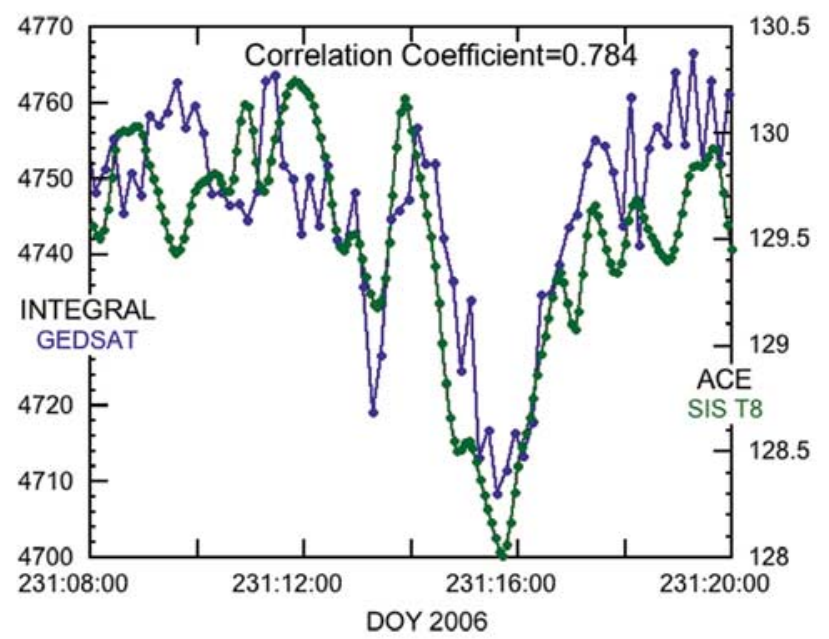

Figure 7. This plot is similar in format to that of Figure 3. (left) The INTEGRAL/GEDSAT and the ACE/SIS T8 count rates as a function of time during the time period shown by the first highlighted box in Figure 2. (right) The same count rates with the ACE measurements delayed by the solar wind propagation time from ACE to INTEGRAL with a correlation coefficient of 0.784 . 


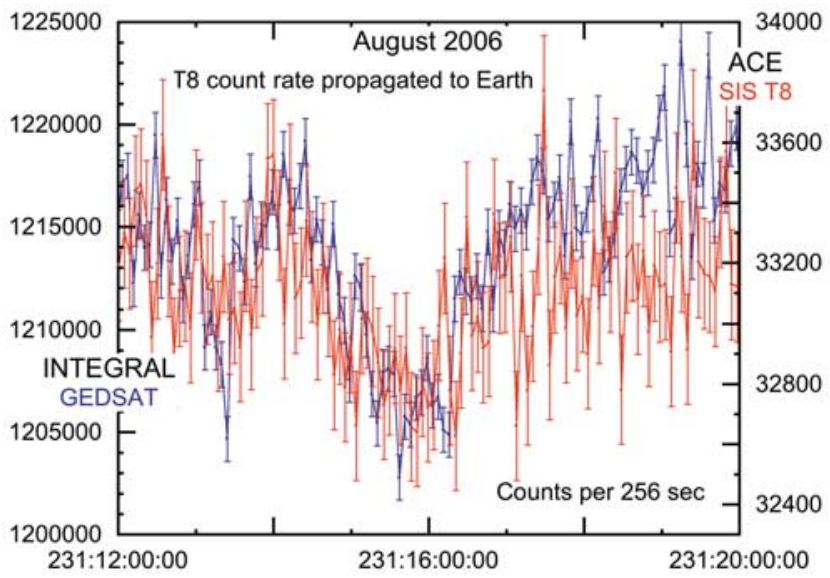

Figure 8. The count rates, 256-s sums, during the latter part of the time period shown in Figure 7 as seen at ACE (SIS T8) at L1 and INTEGRAL (GEDSAT) at Earth, are plotted together. The ACE count rate has been time delayed by the propagation time to Earth using the measured solar wind parameters. The vertical scales of both count rates have been adjusted to make them overlay.

a discontinuity (D). The two shocks are driven by interplanetary coronal mass ejections (ICMEs), which are slightly faster than the ambient solar wind. The discontinuity (D) marks the ICME trailing edge boundary and a return to the ambient solar wind conditions. Although the Forbush decrease on 20 August 2006 is the largest intensity change at both Polar and INTEGRAL during this active time, there are many others on a variety of time scales and amplitudes. The two smaller intensity changes discussed in section 3 are highlighted by rectangles. Note that the first of these highlighted areas occurs just after the shock labeled S2. This region of high magnetic field variance (and hot plasma) corresponds to the magnetosheath of the ICME labeled FR1. It is within this region that the small decrease

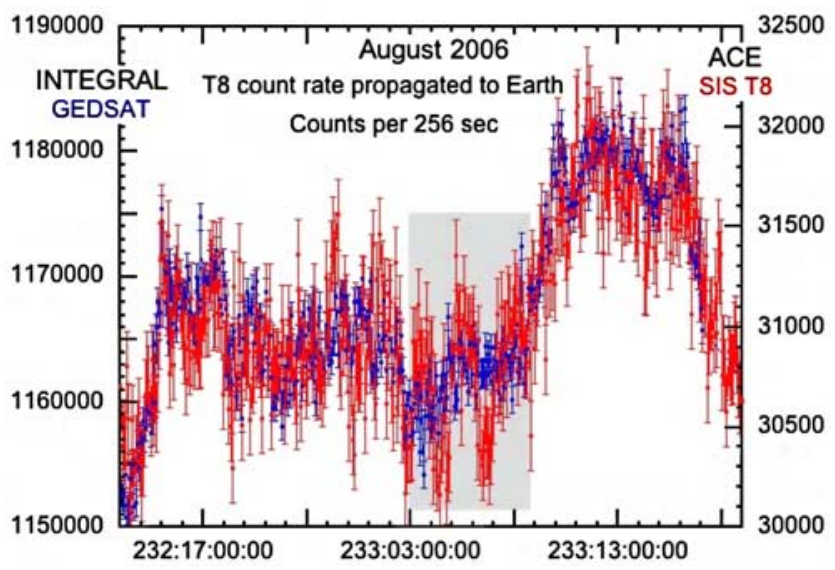

Figure 9. The count rates seen at ACE (SIS T8) and INTEGRAL (GEDSAT) are plotted for the time period delineated by the second highlighted box in Figure 2 (the green box). The ACE count rate has been time delayed by the propagation time to Earth using the measured solar wind parameters. The vertical scales of both count rates have been adjusted to make them overlay.

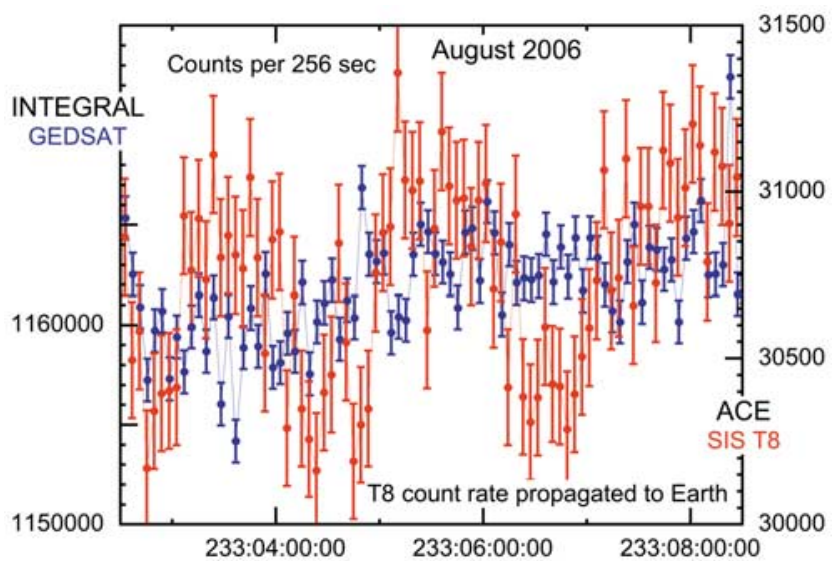

Figure 10. The count rates seen at ACE (SIS T8) and INTEGRAL (GEDSAT) are plotted on an expanded scale for the time period indicated by the gray box in Figure 9, showing the oscillatory behavior of the ACE count rate during this time period.

in the GCR flux studied in section 3 is observed. The Forbush decrease that follows shortly thereafter is associated with the ICME flux-rope-like signature FR1. Immediately following the first flux rope is a larger flux rope signature FR2. It is at the trailing edge of this rope that the rise in count rate associated with latter of the two intervals chosen for study is observed.

[32] Figure 12 shows the relative location of Polar and INTEGRAL near and within the Earth's magnetosphere while the Earth encounters the FD. During this time, Polar passes through the southern polar region on open field lines at $6 \mathrm{Re}$ while INTEGRAL is near apogee at nearly $20 \mathrm{Re}$ above the Earth in the Northern Hemisphere. The ACE spacecraft is located at the L1 Lagrangian point approximately 225 Re sunward of the Earth, and Wind is located at nearly the same radial distance as ACE, but at $100 \mathrm{Re}$ on the dawn side of the Earth.

[33] Note that only minor differences exist between the magnetic field observed by ACE and Wind despite their separation, which means that the scale size of the solar wind magnetic structures is greater than $100 \mathrm{Re}$, much greater than the separation distance between Polar and INTEGRAL and typically far greater than the gyroradius of a $200-\mathrm{MeV}$ proton in the solar wind, the lowest GCR proton energy observed by INTEGRAL (see Figures 13 and 14 (ninth panels)).

[34] A closer look at the solar wind magnetic field and plasma during the first of the two highlighted intervals reveals that distinct regions are visible inside the ICME. Figure 13 shows Wind and ACE interplanetary magnetic field (IMF) and plasma data for 19 August 2006. The first seven panels are the IMF components and scalar field, followed by the plasma velocity, temperature, and density. The eighth panel shows the Wind 3DP suprathermal electron heat flux (strahl) pitch angle distributions, and following that is a running measure of the gyroradius of a $200-\mathrm{MeV}$ proton (the threshold of GEDSAT aboard INTEGRAL) using both ACE and Wind magnetic field. Note that at this resolution, slight differences between ACE and Wind magnetic field and plasma data become apparent, 


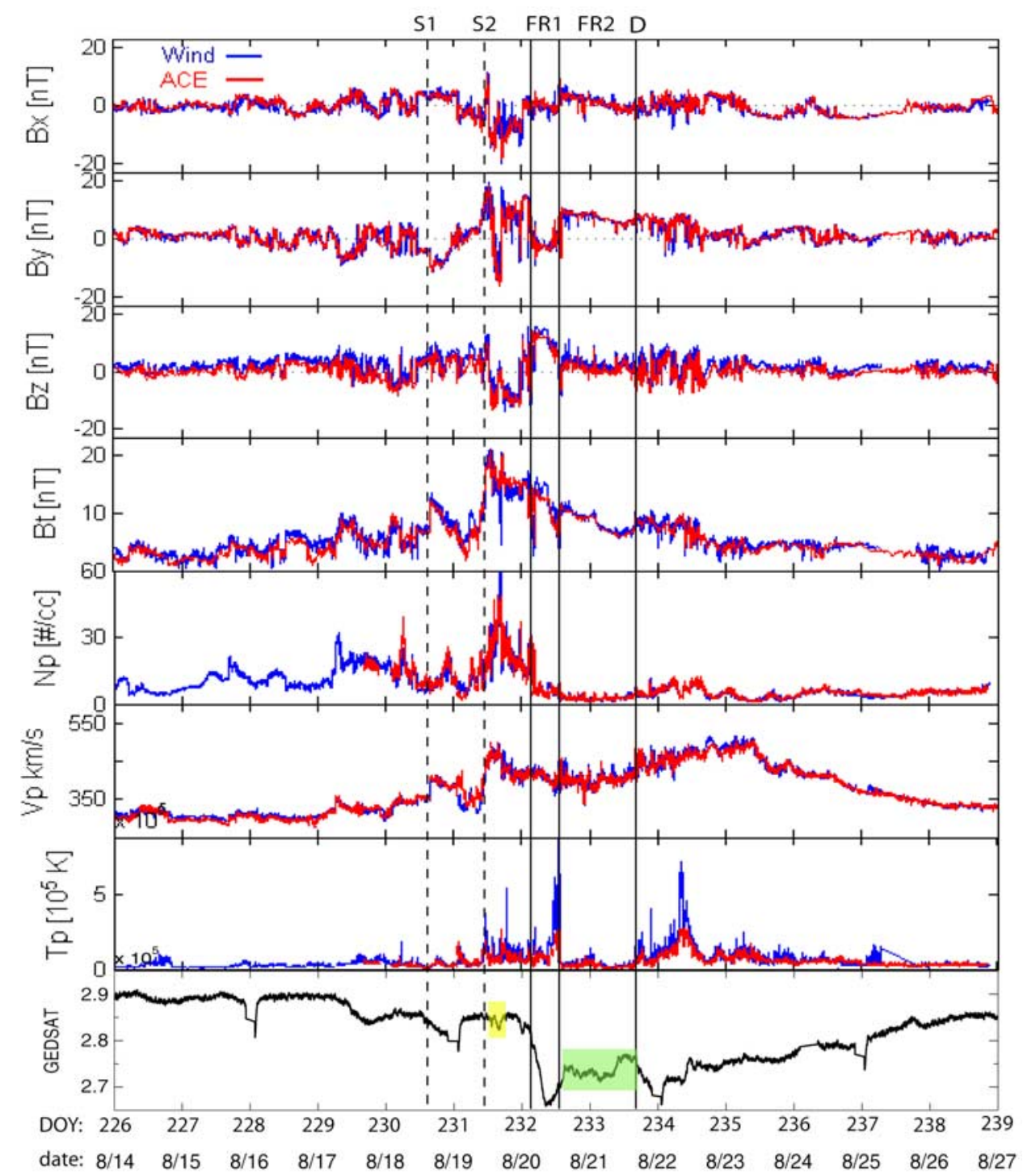

Figure 11. Solar wind magnetic field and energetic particle data during a 2-week interval bracketing the Forbush decrease. The first four panels show the GSE $B_{x}, B_{y}$, and $B_{z}$ components and the scalar field $\left(B_{t}\right)$, respectively, from both ACE (red) and Wind (blue). The fifth to seventh panels show the proton density, velocity, and temperature in the same fashion. The GEDSAT count rate from INTEGRAL is plotted in the last panel. Note there are two interplanetary shocks present during this period (S1 and S2) and a discontinuity (D). The shocks are labeled by dashed vertical lines. Solid vertical lines mark the boundaries of the flux rope type ICME regions FR1 and FR2. The highlighted regions correspond to those in Figure 2.

but since the spacecraft are separated by over $100 \mathrm{Re}$, these differences are relatively minor. Finally, the last panel of Figure 13 contains INTEGRAL and Polar GCR, the INTEGRAL data shifted in time by a $\delta \mathrm{t}$ of $1 \mathrm{~h}$, as determined in section 3 , and with standard $1 \sigma$ error bars showing that some of the large fluctuations are correlated and larger than statistical error.

[35] Nearly simultaneously with the shock S2 passing ACE at approximately 1030 UT, the GCR intensity shows a transient increase at both INTEGRAL and Polar. Following this short increase, the GCR flux intensity decreases. Shortly after the discontinuity D1 (clearly seen as a rotation on the $B_{y}$ component of the field and a drop in field intensity) passes the spacecraft, there is a substantial drop in GCR flux, especially at INTEGRAL. This depression in the GCR flux recovers slightly, but remains depressed relative to the preshock levels during the entire interval until the second discontinuity has passed (D2). At this time, the GCR intensity begins to recover at both spacecraft, but never fully recovers at Polar. Note that the GCR intensity fluctuations at INTEGRAL and Polar are similar early on 19 August, but then diverge after the $B_{z}$ component of the field turns and remains southward (near D2). This shows that the scale size of the GCR variability is on the order or less than the spacecraft separation distance of $25 \mathrm{Re}$ once $B_{z}$ turns southward. This loss of correlation between Polar and INTEGRAL may be indicative of the 

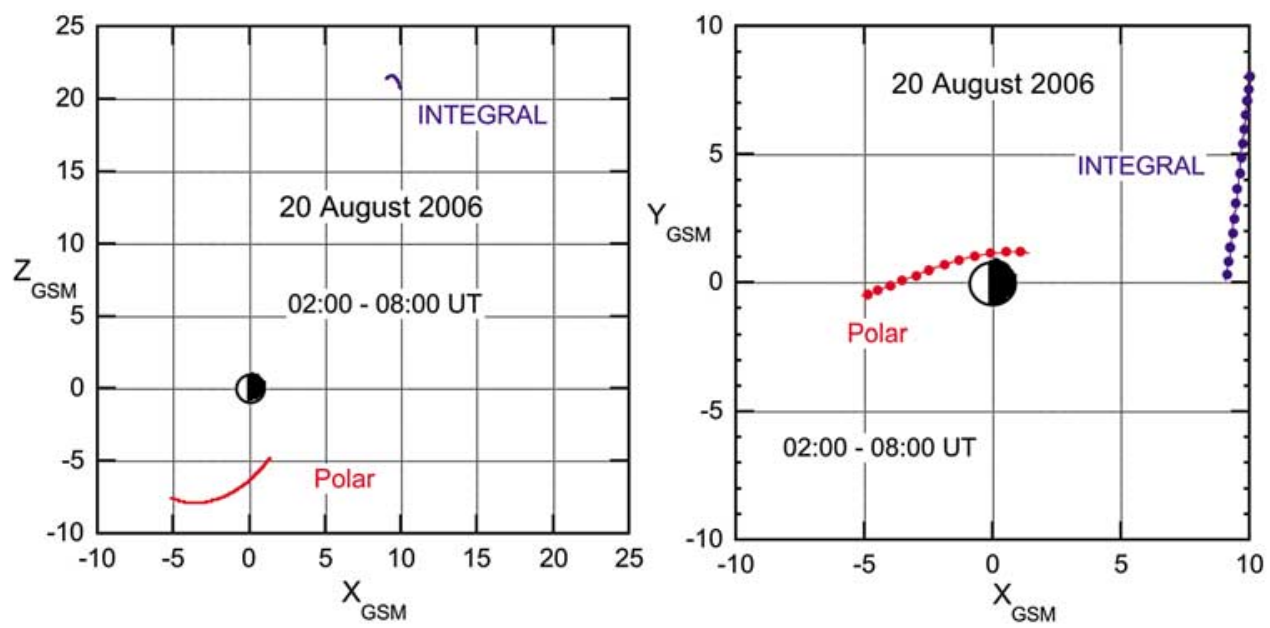

Figure 12. Location of Polar and INTEGRAL within the Earth's magnetosphere (left) in the meridional plane and (right) in the equatorial plane during the onset of the Forbush decrease from 0200 to 0800 UT. Note that Polar passes through the southern polar region on open field lines at 6 Re while INTEGRAL is near apogee at nearly $20 \mathrm{Re}$ above the Earth in the Northern Hemisphere.

change from a quiet to an active magnetosphere during this time.

[36] Interestingly, the electron heat flux shows different flux tube connectivity, also associated with the different regions of the ICME shown in Figure 13. That energetic particle intensities commonly undergo dispersionless modulation in direct association with discontinuous changes in the solar wind electron strahl is not altogether a new concept, especially in association with "impulsive" solar flares [Gosling et al., 2004]. Mazur et al. [2000] and Giacalone et al. [2000] also discussed the concept that dropouts in energetic ion fluxes from impulsive flares as observed at the Earth may be due to the mixing of empty and populated flux tubes as the these tubes convect out in the solar wind. However, the idea that intensity levels in the omnidirectional GCR flux show a similar partitioning because of the magnetic field regions is a new observation. Studies by Burlaga et al. [1993] and Burlaga and Ness [1998] have demonstrated that cosmic ray intensity is generally anticorrelated with interplanetary magnetic field strength on a variety of scales. This third possibility, besides turbulence and topology changes, could be causing the observed variability in the GCR flux, and will be examined in a future publication.

[37] Moving on to the time period containing the second highlighted region on 21 August 2006 reveals yet another intensity change, seemingly correlated with the different regions within the ICME. Figure 14 shows the solar wind parameters in a fashion similar to Figure 13 but for 21 August 2006. Again, the IMF and solar wind plasma data are displayed, followed by the Wind 3DP electron heat flux, the running gyroradius of a $200 \mathrm{MeV}$ proton, and the time-shifted GCR flux at INTEGRAL. Again, there are slight differences between ACE and Wind data, but these differences are minor compared to the $100 \mathrm{Re}$ separation between the spacecraft.

[38] From Figure 14, the highlighted interval begins while the spacecraft are within the latter portion of the flux rope ICME (FR2). As in Figure 13, distinct regions of the
ICME are apparent at this resolution. The discontinuities labeled by the vertical dashed lines delineate one such region within the flux rope (FR2). Note that the GCR flux at INTEGRAL begins to increase around $0730 \mathrm{UT}$, reaching a steady state maximum at $\sim 0900$ UT, just after the first discontinuity passes the spacecraft. The discontinuity is most easily observed in the $B_{y}$ and $B_{z}$ components of the magnetic field. A second discontinuity at $\sim 1545$ UT marks the end of the ICME, shown by the rotations in the $B_{y}$ and $B_{z}$ components, a change in the scalar field, and an increase in proton temperature to more typical solar wind values. Once this second discontinuity passes the spacecraft, the GCR begins to decrease. However, the amount of the decrease is difficult to ascertain as INTEGRAL passes through perigee near the end of the day.

[39] Interestingly, the electron heat flux again shows different flux tube connectivity associated with the different regions of the ICME. Note that a nearly isotropic electron distribution in the latter part of the flux rope region may indicate a trapped population. Entry into a region of antisunward, unidirectional electron streaming (indicated by the cold, intense electron beam at nearly $0^{\circ}$ ) is nearly simultaneous with the rise in GCR flux intensity. At the end of the ICME, the field-aligned beam is still present, although the distribution turns more toward a bidirectional streaming signature on heated flux tubes. This region is also coincident with the beginning of GCR decrease.

\section{Conclusions}

[40] Since their discovery in the late 1930s, Forbush decreases in GCR intensity have been repeatedly put into context with increasingly detailed observations of CMEs and in situ observations of the solar wind and energetic particles. In this paper, we have focused upon smaller amplitude and higher-frequency ( $\mathrm{mHz}$ range) variability in the GCR intensity than is described by a classic Forbush decrease. Measuring such small amplitude variability in the GCR is an experimental challenge requiring very 


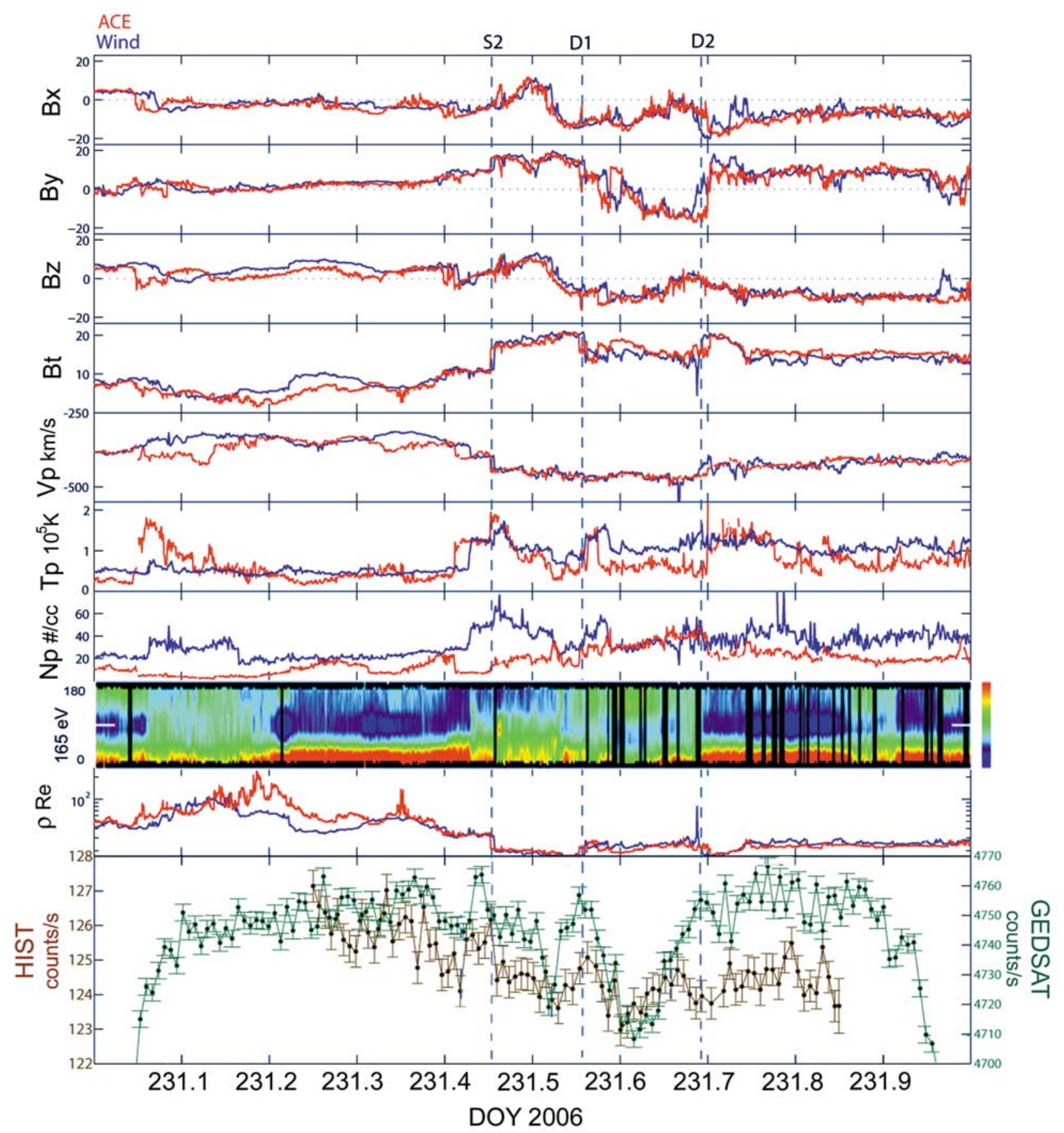

Figure 13. ACE and Wind IMF and plasma data for 19 August 2006 are shown. The first four panels are the IMF components and scalar field, followed by the plasma velocity, temperature, and density from both ACE (red trace) and Wind (blue trace). The eighth panel shows the Wind 3DP suprathermal electron heat flux (strahl) pitch angle distributions, and following that is a running measure of the gyroradius of a $200-\mathrm{MeV}$ proton (the threshold of the GEDSAT aboard INTEGRAL) using both ACE and Wind magnetic field. The last panel contains INTEGRAL and Polar GCR count rates with error bars showing the $1 \sigma$ error. Both INTEGRAL and Polar data have been time shifted with $\delta \mathrm{t}=1 \mathrm{~h}$, as determined in section 3 .

large instrumental geometric factors to make statistically significant measurements in time periods of a few minutes or less. Only by using the unique spacecraft instrumentation and the highest data rate acquisition methods available aboard Polar, INTEGRAL, and ACE have we been able to undertake such a study.
[41] Focusing upon the GCR intensity within a 3-day interval during an unusually active period in the solar wind from 19 August 2006 to 21 August 2006 reveals many intensity variations in the GCR on a variety of time scales and amplitudes. As shown in Figure 2, these intensity variations, especially in the highlighted regions, are greater 


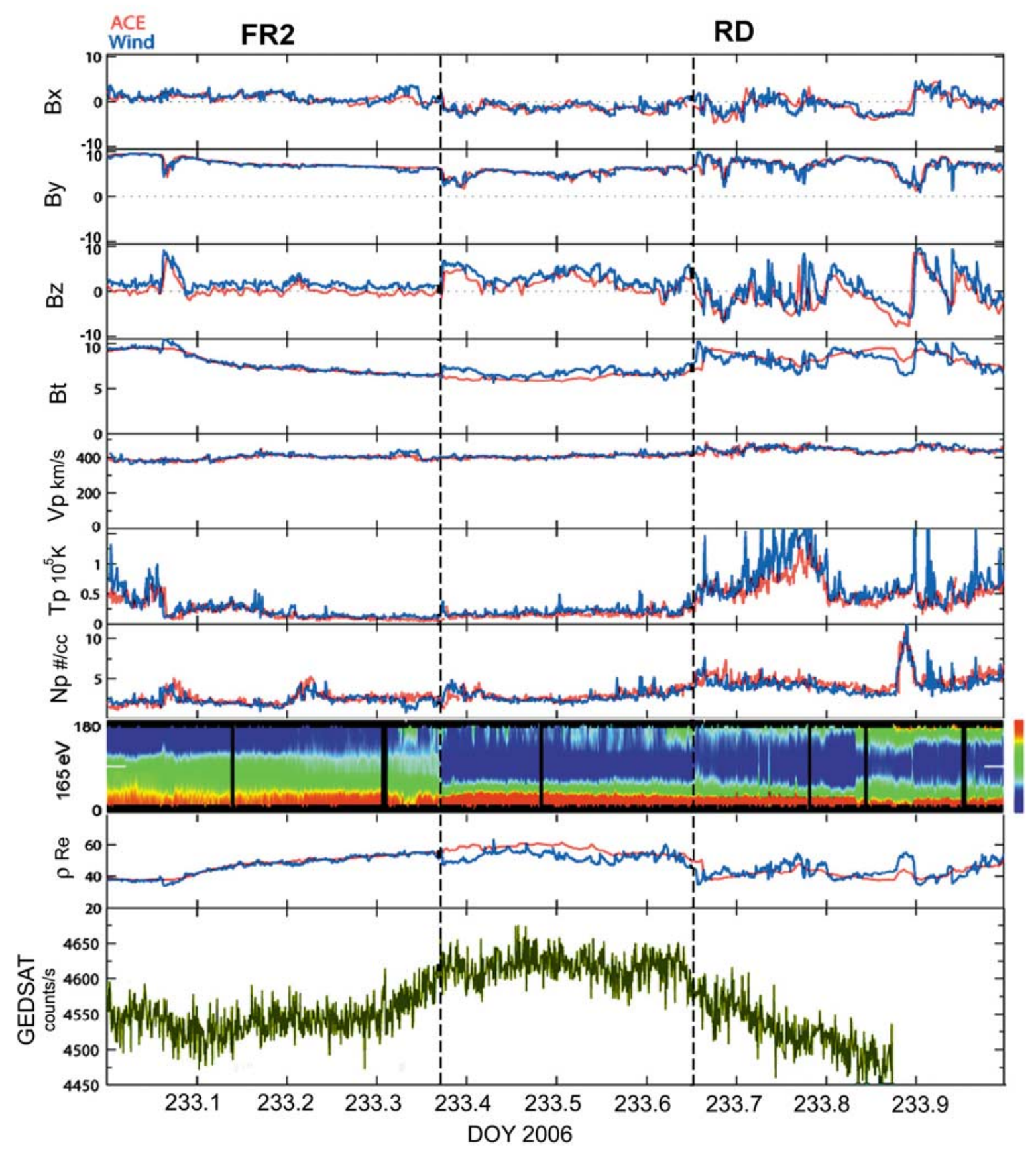

Figure 14. ACE and Wind solar wind parameters are shown in a fashion similar to Figure 13 but for 21 August 2006. The first seven panels contain the IMF and solar wind plasma data from both ACE (red trace) and Wind (blue trace), followed by the Wind 3DP electron heat flux, the running gyroradius of a $200 \mathrm{MeV}$ proton, and the GCR intensity count rate at INTEGRAL (time shifted with $\delta \mathrm{t}=1 \mathrm{~h}$, as determined in section 3).

than the $3 \sigma$ error in all the data sets used. Note that the time history profiles at ACE, INTEGRAL, and Polar are very similar during many of the variations. Considering that the solar wind changes only about $100 \mathrm{~km} / \mathrm{s}$ during these time periods, it seems clear that parcels of solar wind moving outward are transporting the GCR population and the fine structure in GCR intensities along with the Forbush decrease are propagated outward from ACE to the Earth with very little change. This solar wind convection of GCR fine structure is observed for both increases in GCR intensity and decreases, which indicates that the shortperiod variability is not due solely to changes in the GCR population on a large scale, but is also due to small-scale intensity structures being transported outward by the solar wind. Putting these particle observations in context with both large-scale and small-scale solar wind transient 
structures during this period, it is clear that the GCR fine structure decreases are associated with ICME magnetosheath regions of high magnetic field variance and changes in field magnitude just behind interplanetary shocks, while the Forbush decrease is associated with the flux rope interior of the ICME. Most notably, fine structure increases and decreases are bracketed by solar wind magnetic field discontinuities, clearly seen as discontinuous rotations of the field components at ACE and at Wind, which sometimes accompany changes in field intensity.

[42] Interestingly, the electron heat flux shows different flux tube connectivity also associated with the different regions of the ICME and magnetosheath. Gosling et al. [2004] first discussed the idea that solar energetic particle intensities commonly undergo dispersionless modulation in direct association with discontinuous changes in the solar wind electron strahl. The observations in this paper show that the intensity levels in the GCR flux undergo a similar partitioning, possibly because of the different magnetic field regions having differing magnetic topologies. This new observation of GCR intensity being modulated by the local magnetic field topology will be explored in further detail in a future publication. Future studies will include determining whether turbulence [Mazur et al., 2000; Giacalone et al., 2000], magnetic topology [Gosling et al., 2004; Richardson et al., 2000; Vasquez et al., 2001], magnetic field strength [Burlaga et al., 1993; Burlaga and Ness, 1998] or a combination of these properties in the solar wind are the cause of these variations in the GCR flux intensity. Such comparisons will lead to further understanding of the underlying physics of energetic particle transport though the interplanetary medium.

[43] Acknowledgments. This work was supported under the Aerospace Corporation's Independent Research and Development Program. The work at Caltech was supported by NASA under grant NNX08AI11G. The authors would like to thank the INTEGRAL team for their assistance and for supplying the INTEGRAL data.

[44] Amitava Bhattacharjee thanks the reviewers for their assistance in evaluating this paper.

\section{References}

Bieber, J. W., and P. Evenson (1995), Spaceship Earth-An optimized network of neutron monitors, Conf. Pap. Int. Cosmic Ray Conf. 24th, 4, 1316-1319.

Blake, J. B., et al. (1995), CEPPAD: Comprehensive energetic particle and pitch angle distribution experiment on Polar, Space Sci. Rev., 71, 531562.

Burlaga, L., and N. Ness (1998), Magnetic field strength distributions and spectra in the heliosphere and their significance for cosmic ray modulation: Voyager 1, 1980-1994, J. Geophys. Res., 103, 29,719-29,732.

Burlaga, L., F. McDonald, and N. Ness (1993), Cosmic ray modulation and the distant heliospheric magnetic field: Voyager 1 and 2 observations from 1986 to 1989, J. Geophys. Res., 98, 1-11.

Cane, H. V. (2000), Coronal mass ejections and Forbush decreases, Space Sci. Rev., 93, 55-77.

Contos, A. R. (1997), Complete description and characterization of the high sensitivity telescope (HIST) onboard the Polar satellite, M.S. thesis, Boston Univ, Boston, Mass.
Giacalone, J., J. R. Jokipii, and J. E. Mazur (2000), Small-scale gradients and large-scale diffusion of charged particles in the heliospheric magnetic field, Astrophys. J., 532, L75-L78, doi:10.1086/312564.

Gosling, J. T, R. M. Skoug, D. J. McComas, and J. E. Mazur (2004), Correlated dispersionless structure in suprathermal electrons and solar energetic ions in the solar wind, Astrophys. J., 614, 412-419.

Hajdas, W., P. Bühler, C. Eggel, P. Favre, A. Mchedlishvili, and A. Zehnder (2003), Radiation environment along the INTEGRAL orbit measured with the IREM monitor, Astron. Astrophys., 411, L43-L47.

Jean, P., et al. (2003), SPI instrumental background characteristics, Astron. Astrophys., 411, L107-L112.

Kuwabara, T., J. W. Bieber, and R. Pyle (2008), Heliospheric physics with IceTop, Conf. Pap. Int. Cosmic Ray Conf. 30th, 1, 339-342.

Lockwood, J. A. (1971), Forbush decreases in the cosmic radiation, Space Sci. Rev., 12, 658-715.

Mazur, J. E., G. M. Mason, J. R. Dwyer, J. Giacalone, J. R. Jokipii, and E. C. Stone (2000), Interplanetary magnetic field line mixing deduced from impulsive solar flare particles, Astrophys. J., 532, L79-L82.

McCracken, K. G., U. R. Rao, and R. P. Bukata (1966), Recurrent Forbush decreases associated with $M$-region magnetic storms, Phys. Rev. Lett., 17, $928-932$

Mulligan, T., et al. (2008), Unusual solar energetic proton fluxes at $1 \mathrm{AU}$ within an interplanetary CME, Conf. Pap. Int. Cosmic Ray Conf. 30th, 1, $179-182$

Munakata, K., S. Yasue, C. Kato, J. Kota, M. Tokumaru, M. Kojima, A. A. Darwish, T. Kuwabara, and J. W. Bieber (2006), On the cross-field diffusion of galactic cosmic rays into the magnetic flux rope of a CME, in Advances in Geosciences, vol. 2, edited by W.-H. Ip and M. Duldig, pp. 115-124, World Sci, Hackensack, N. J.

Ni, W.-T., et al. (2006), ASTRID and ASTROD I: Progress report, J. Phys. Conf. Ser., 32, 154-160.

Richardson, I. G. (2004), Energetic particles and corotating interaction regions in the solar wind, Space Sci. Rev., 111, 267-376.

Richardson, I. G., G. Wibberenz, and H. V. Cane (1996), The relationship between recurring cosmic ray depressions and corotating solar wind streams at $\leq 1$ AU: IMP 8 and Helios 1 and 2 anticoincidence guard rate observations, J. Geophys. Res., 101, 13,483-13,496.

Richardson, I. G., V. M. Dvornikov, V. E. Sdobnov, and H. V. Cane (2000), Bidirectional particle flows at cosmic ray and lower $(\sim 1 \mathrm{MeV})$ energies and their association with interplanetary coronal mass ejections/ejecta, J. Geophys. Res., 105, 12,579-12,591.

Stone, E. C., et al. (1998), The solar isotope spectrometer for the Advanced Composition Explorer, Space Sci. Rev., 86, 357-408.

Sumner, T. J., and D. N. A. Shaul (2004), The observation of gravitational waves from space using LISA, Mod. Phys. Lett. A, 19, 785-800.

Teegarden, B. J., et al. (1997), SPI: A high resolution imaging spectrometer for INTEGRAL, in Proceedings of the Fourth Compton Symposium, edited by C. D. Dermer, M. S. Strickman, and J. D. Kurfess, AIP Conf. Proc., 410, 1535-1543.

Vasquez, B. J., C. J. Farrugia, S. A. Markovskii, J. V. Hollweg, I. G. Richardson, K. W. Ogilvie, R. P. Lepping, R. P. Lin, and D. Larson (2001), Nature of fluctuations on directional discontinuities inside a solar ejection: WIND and IMP 8 observations, J. Geophys. Res., 106, 29,28329,298 .

Vedrenne, G., et al. (2003), SPI: The spectrometer aboard INTEGRAL, Astron. Astrophys., 411, L63-L70.

J. B. Blake and T. Mulligan, Aerospace Corporation, Los Angeles, CA 90009, USA. (tamitha.mulligan@aero.org)

M. Galametz, Laboratoire AIM, DSM, CEA, Université Paris Diderot, DAPHNE, Service d'Astrophysique, CNRS, F-91191 Gif-sur-Yvette, France.

R. A. Leske and R. A. Mewaldt, California Institute of Technology, Pasadena, CA 91125, USA.

J. J. Quenby and D. Shaul, High Energy Physics Group, Blackett Laboratory, Imperial College London, Prince Consort Road, London SW7 2BZ, UK 